\title{
Toward Flexible Piezoelectrets with High Environmental Stability: A Hybrid Approach
}

\author{
Zhe Liu, ${ }^{1,2}$ Min Zeng, ${ }^{3}$ Hui Wang, ${ }^{1,2}$ Xiaolin Wang, ${ }^{1,2}$ Yan $\mathrm{Li}^{1,2}$ and Changchun Zeng ${ }^{1,2,{ }^{*}}$
}

\begin{abstract}
In this work, an effective and low-cost method to fabricate hybrid piezoelectrets is based on cyclic olefin copolymer (COC) and polypropylene (PP). The electromechanical properties of the hybrid piezoelectrets were characterized. In the hybrid piezoelectrets, PP constituted the overall structure while COC was used as surface layers for charge storage. The hybrid piezoelectrets exhibited good piezoelectric properties, flexibility, and significantly improved environmental stability. The hybrid piezoelectrets had a piezoelectric coefficient $d_{33}$ up to $1200 \mathrm{pC} / \mathrm{N}$. The PP piezoelectrets lost all piezoelectric activity after annealing for 16 hours at $70{ }^{\circ} \mathrm{C}$. By comparison, even after 200 hours of annealing at $110{ }^{\circ} \mathrm{C}$, the hybrid COC/PP piezoelectrets still retained $25 \%$ of the activity. The hybrid piezoelectrets also showed excellent stability under high humidity. The performance of the hybrid piezoelectrets as force sensors were demonstrated.
\end{abstract}

Keywords: Piezoelectrets; Piezoelectric activity; Environmental stability; Cyclic olefin copolymer; Polypropylene; Sensors. Received: 06 October 2021; Revised: 04 November 2021; Accepted: 06 December 2021.

Article type: Research article.

\section{Introduction}

Piezoelectric materials are widely used in sensing as they generate electric signals when under stress. ${ }^{[1,2]}$ Piezoelectric ceramic is one of the most widespread traditional piezoelectric materials that show excellent thermal stability over broad temperature ranges. ${ }^{[3]}$ Piezoelectric polymers have also been widely studied because of their advantages, such as being nontoxic, flexible, lightweight, and mass-production benign. These are particularly valuable to portable and wearable devices and for new applications requiring flexible sensors. ${ }^{[4]}$ With the rapid advancement of soft robotics, soft skin, and wearable devices, polymer-based flexible sensors have attracted a great deal of attention. ${ }^{[5]}$ But, one issue with piezoelectric polymer sensors is their low piezoelectric activity. Piezoelectrets (also commonly known as piezoelectrets) are polymer-based porous thin films exhibiting pseudo piezoelectric properties resulting from the space charges within the pores (voids or cavities). Not only even lighter, but the piezoelectrets also showed substantially higher

\footnotetext{
${ }^{1}$ High-Performance Materials Institute, Florida State University,

Tallahassee, FL, 32310, USA.

${ }^{2}$ Department of Industrial and Manufacturing Engineering, FAMU-

FSU College of Engineering, Tallahassee, FL, 32310, USA.

${ }^{3}$ Nanjing Polytechnic Institute, No.625, Geguan Road, Dachang,

Luhe District, Nanjing City, Jiangsu Province, 210048, China.

*Email: zeng@eng.famu.fsu.edu (C. Zeng)
}

piezoelectric activities. Their potential applications have been widely reported in sensors, actuators, novel biomedical devices, pressure mapping, piezoelectric motors, and ultrasonic transducers. ${ }^{[6]}$

Unlike piezoelectric ceramics and polymers, piezoelectricity in piezoelectrets results from macroscopic dipoles instead of molecular dipoles, by dielectric barrier discharge within the pores under a strong electric field. ${ }^{[1,2]}$ Because of the dielectric properties of polymers, positive and negative charges are stored in the internal and opposite surfaces of the polymer cells after the discharge,,$^{[7,8]}$ leading to the generation of macroscopic dipoles. The piezoelectricity depends on 'dipole' density, which varies under applied stress or electric field. ${ }^{[9]}$ The electromechanical properties, charge density and distributions, charge storage properties, and timetemperature stability have been investigated. ${ }^{[1,10,11]}$

Polypropylenes (PPs) are the most commonly used materials for piezoelectrets, which have been produced commercially. ${ }^{[12]}$ Polypropylenes owe their popularity to their low cost, good flexibility, and adequate charge storage capability. However, they suffer low thermal stability and have poor charge stability at elevated temperatures. The maximum working temperature of PP piezoelectrets is lower than $60{ }^{\circ} \mathrm{C},{ }^{[13,14]}$ and the piezoelectric properties of the PP piezoelectrets are strongly affected by temperature. ${ }^{[15]}$ Therefore, much effort has been made to improve the maximum working temperature of PP piezoelectrets. 
Approaches have included chemical modifications by oxidation and fluorination, ${ }^{[16-18]}$ treatment of PP films by repeated expansions, ${ }^{[19-22]}$ and the use of cross-linked PP. ${ }^{[21,23]}$ Other studies in piezoelectrets with improved thermal stability and working temperature have focused on using other polymers with good insulating and better charge-storage properties than $\mathrm{PP}$ at elevated temperatures, including polytetrafluoroethylene (PTFE), ${ }^{[13,24-26]}$ polycarbonate (PC), ${ }^{[27]}$ and cyclic olefin copolymers (COCs). ${ }^{[15,28]}$

COCs are promising candidate polymers because of their better thermal stability than PPs. ${ }^{[2,30]}$ COCs also exhibit excellent charge-storage stability, good environmental stability, low water absorption, low dielectric constant, and good mechanical properties. ${ }^{\left[{ }^{11]}\right.}$ COCs based piezoelectrets with high piezoelectric activity were reported previously. ${ }^{[15]}$ However, COCs are more expensive and require substantially more challenging processing conditions. In addition, COCs of high thermal stability are stiff and brittle, detrimental to their applications as flexible sensors.

In this work, we discuss the development of COC/PP hybrid piezoelectrets. The hybrid piezoelectrets combine the advantages of both components, namely, COC's superior charge-storage properties and thermal stability and PP's good flexibility and low cost. The design, fabrication, and characterization of the hybrid piezoelectrets were discussed. Their potential use as force sensors were also demonstrated.

\section{Experimental}

\subsection{Materials}

PP films with a thickness of $30 \mu \mathrm{m}$ were purchased from the Goodfellow Cambridge Ltd. High-temperature grade COC pellets, and COC films (Topas 6017) with a glass transition temperature at $180{ }^{\circ} \mathrm{C}$ were from Topas Advanced Polymers. Cyclohexane (anhydrous, 99.5\%) was obtained from SigmaAldrich.

\subsection{Fabrication of hybrid COC/PP piezoelectret 2.2.1 Overall structure}

The hybrid COC/PP piezoelectrets consisted of five PP layers, as shown in Figs. 1 and 2. Of the five layers, three (layers 1, 3, 5) were solid films, and the other two (layers 2,4 ) were patterned films with rectangular cavities with designed dimensions fabricated using a $\mathrm{CO}_{2}$ laser (VersaLASER, Universal Laser Systems). These cavities had the same dimensions but were offset by half the cavity width. Furthermore, Layers 1 and 5, the top and bottom PP layers, were coated with $\mathrm{COC}$ on one side, whereas the other three middle layers were coated with $\mathrm{COC}$ on both sides. The coating process is described in section 2.2 .2 below. Fig. $2 b$ illustrates the architecture and material placement of the porous films. Control samples were also fabricated with pure COC films. The control sample had the same geometric dimensions as the hybrid piezoelectrets.

\subsubsection{Preparation of COC/PP hybrid film}

COC-coated PP films were fabricated with a scalable doctor blade coating technology. COC pellets were dissolved in cyclohexane solvent to obtain a solution with a solids content of $10 \%$ by weight. PP substrates were cleaned with acetone followed by deionized water and dried. The $\mathrm{COC} /$ cyclohexane solution was coated on a PP substrate film using a doctor blade. Coatings were dried at room temperature for 24 hours to allow solvent evaporation.

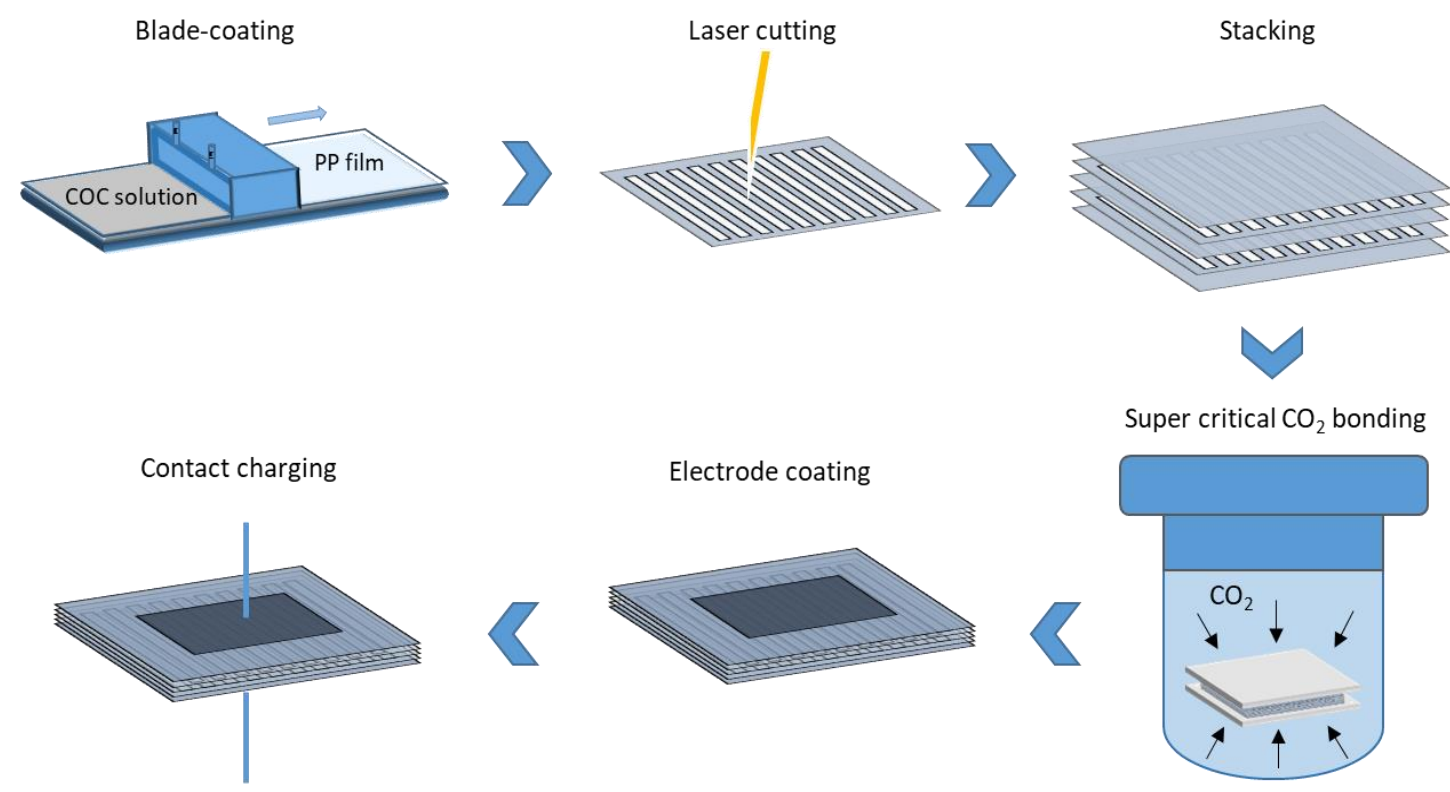

Fig. 1 Schematic of fabrication of COC/PP hybrid piezoelectrets. Three PP films were coated with COC by blade coating. Of the three films, one was coated on both sides sequentially. At the same time, two PP films were machined with rectangular cavities by laser cutting. There were subsequently also coated by $\mathrm{COC}$ on both sides. The layers stack was bonded together by carbon dioxide assisted assembled to form the porous assemblage. The assemblage was charged by contact charging and resulted in the COC/PP hybrid piezoelectret. 


\subsubsection{Assembly of COC/PP piezoelectrets}

The hybrid piezoelectrets were fabricated by bonding together the five-layer COC/PP films using a supercritical carbon dioxide $\left(\mathrm{CO}_{2}\right)$-assisted assembly process, which allows the fusion of the COCs while minimally perturbing the overall structure. ${ }^{[15]}$ The film stack was placed in a high-pressure vessel (CL-1, High-Pressure Equipment Company), and carbon dioxide was injected by a high-pressure ISCO syringe pump (500 HP, Teledyne Technologies, Inc.). The system was heated and maintained at $120^{\circ} \mathrm{C}$ with a constant $\mathrm{CO}_{2}$ pressure of $10 \mathrm{MPa}$ for $12 \mathrm{~h}$, after which the pressure was slowly released, and the assembled samples were removed from the pressure vessel.

\subsubsection{Charging of COC/PP piezoelectrets}

The assembled structures were sputter-coated with platinum $(\mathrm{Pt})$ as the electrodes and charged in the air at room temperature. Direct-contact charging using a precision highvoltage power supply (PNC 10000-6ump, Heinzinger electronic $\mathrm{GmbH}$ ) was conducted at a voltage of $5 \mathrm{kV}$ for 10 s. Afterward, the hybrid COC/PP piezoelectrets were stored between aluminum foils for $12 \mathrm{~h}$ to complete fast relaxation processes.

\subsection{Characterization}

\subsubsection{Morphology}

The structure of the hybrid COC/PP piezoelectric films were studied using optical microscopy and scanning electron microscopy (SEM, JEOL 7401F). Samples for SEM observation were coated with a Pt layer by sputter coating. An accelerating voltage of $10 \mathrm{kV}$ was used to acquire images. The thickness of COC coating layers was determined from the SEM images using ImageJ (National Institutes of Health) at different magnifications.

\subsubsection{Piezoelectric properties}

The piezoelectric $\mathrm{d}_{33}$ coefficients were measured by the quasistatic method using a Keithley electrometer (6514A, Keithley Instruments, Inc.). The piezoelectric $\mathrm{d}_{33}$ coefficient is defined as the equation: $\mathrm{d}_{33}=\mathrm{Q} / \mathrm{F}$, in which $\mathrm{Q}$ and $\mathrm{F}$ are the induced charge and applied force perpendicular to the hybrid COC/PP films. A preload of $1 \mathrm{~N}$ was added first to the top of the electrode to eliminate the air gap effect. A calibrated force $F$ was first applied onto the samples, then removed. The induced charge Q was measured and integrated over $10 \mathrm{~s}$. The average values from five separate measurements were used for calculation.

Hysteresis loop tests were performed to investigate the charge buildup process in the pores (artificial voids, cavities, used interchangeably) of the piezoelectrets. Voltages ramp (1000 to 7000 volts) was applied to the samples (TREK model 609B), and the polarization strength was recorded using RADIANT Precision Premier II.

\subsubsection{Finite Element Analysis}

The unit cell structures for COCs and COC/PP hybrids were generated using the COMSOL Multiphysics Simulations package. The geometries of each structure are shown in Fig. 6. Finite element analysis with compression loading conditions was performed on the two models using the material properties list in Table 1. A linear elastic modulus assumption was used in the simulation.

Table 1. Materials properties.

\begin{tabular}{cccc}
\hline & $\begin{array}{c}\text { Density } \\
\left(\mathrm{kg} \mathrm{m}^{-3}\right)\end{array}$ & $\begin{array}{c}\text { Young's } \\
\text { modulus } \\
(\mathrm{Pa})\end{array}$ & $\begin{array}{c}\text { Poisson's } \\
\text { ratio }\end{array}$ \\
\hline $\begin{array}{c}\text { Polypropylene (PP) } \\
\text { Cyclic olefin copolymer } \\
(\mathrm{COC})\end{array}$ & 900 & $1.5 \times 10^{9}$ & 0.41 \\
\hline
\end{tabular}

\subsubsection{Environmental stability}

The long-term thermal stability of the $d_{33}$ coefficient was studied by isothermal decay. Samples were divided into two groups and were annealed isothermally at $70{ }^{\circ} \mathrm{C}$ and $110{ }^{\circ} \mathrm{C}$, respectively, for 200 hours. They were then cooled to room temperature, and the $d_{33}$ coefficient was measured. The humidity stability of the piezoelectric films was explored using an environmental test chamber. The test chamber system (Electro-tech system, Inc.) was comprised of a sealed acrylic enclosure, a microprocessor humidity controller (ETS Model 5100-240) in conjunction with a dehumidification system (Model 5461) and an ultrasonic humidification system (Model 5462). The piezoelectrets were conditioned in a test chamber at relative humidity (RH) of $90 \%$ for one week and then dried at room temperature for 48 hours before their piezoelectrets coefficient was measured.

\subsubsection{Sensor performance}

As a flexible piezoelectric sensor, the performance of the hybrid COC/PP piezoelectrets was tested by applying cyclic compression forces using a Shimadzu AGS-J with a crosshead velocity of $0.5 \mathrm{~mm} / \mathrm{min}$, and measuring the dynamic voltage outputs were recorded by an electrometer (Kistler 6517A). Data were transmitted to a PC and recorded by LabVIEW software.

\section{Results and discussion}

\section{1 $\mathrm{COC} / \mathrm{PP}$ hybrid piezoelectrets fabrication and} morphology

Hybrid piezoelectrets were fabricated by assembling five layers of COC-coated PP films (Fig. 1, step 3). Layer 1 and 5 have a COC coating layer on one side, and layers 2-4 are double side COC coating, as shown in Fig. 2a. The five layers of films were assembled by supercritical $\mathrm{CO}_{2}$ bonding at 120 ${ }^{\circ} \mathrm{C}$ and $10 \mathrm{MPa} \mathrm{CO}_{2}$ pressure. ${ }^{[15]}$ During bonding, polymer chains undergo wetting, diffusion, and entanglement to form bonded interface. ${ }^{[32]}$ This requires processing temperatures above the glass transition temperatures ( $\mathrm{Tg}$ ) and/or melting temperatures $(\mathrm{Tm})$ of the polymers. Because of the high 


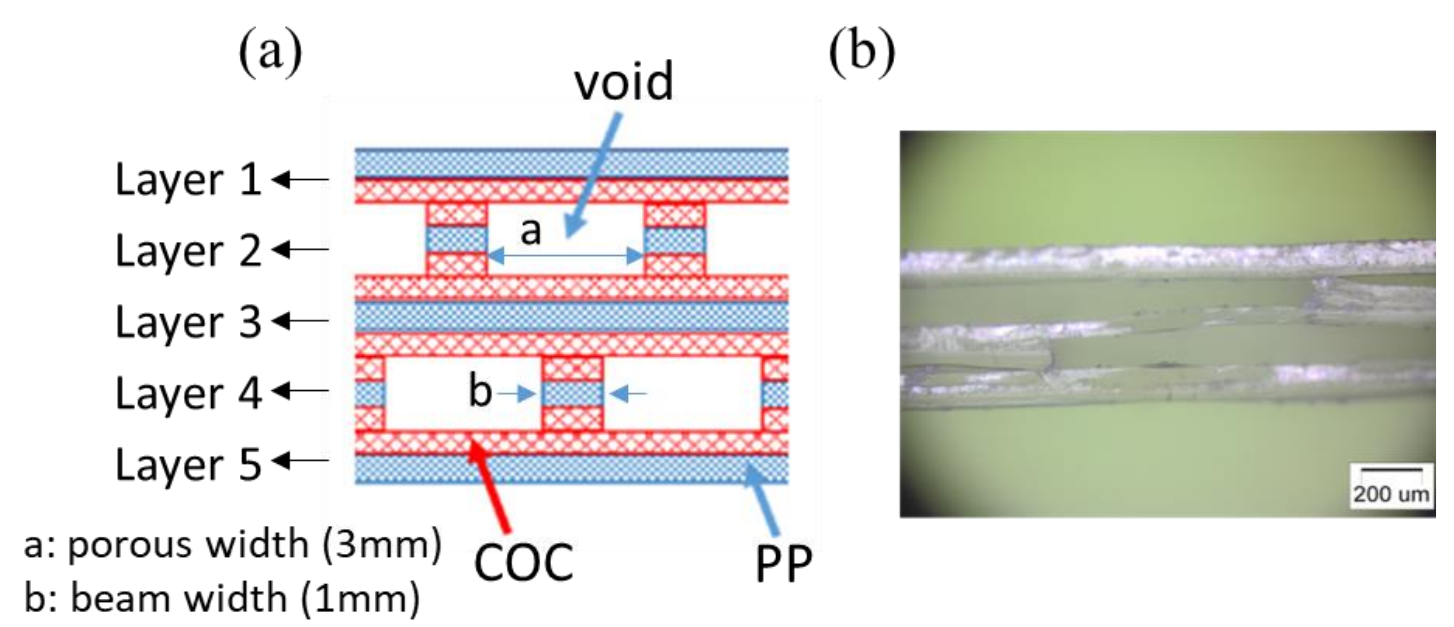

Fig. 2 (a) Schematic of the hybrid COC/PP piezoelectrets with cross-section view showing the construction and composition of the film assembly; (b) optical image of the cross-section for a fabricated hybrid COC/PP piezoelectrets.

solubility of carbon dioxide in COCs and the increased COC polymer chain mobility, ${ }^{[33]}$ the $\mathrm{Tg}$ was significantly reduced. This enables more rapid polymer chain movement and interfacial diffusion for bonded interface development, leading to successful bonding at significantly lower temperatures. In the current process, the bonding temperature was $120{ }^{\circ} \mathrm{C}, 60{ }^{\circ} \mathrm{C}$ below the $\mathrm{Tg}$ of $\mathrm{COC} 6017$, and $40{ }^{\circ} \mathrm{C}$ below the Tm of PPs. Such a low processing temperature minimized the deformation of the structures and greatly improved the structure fidelity of the fabricated piezoelectrets. Fig. 2b shows a cross-section view of assembled hybrid foam, which was observed by optical microscopy.

SEM was used to examine the adhesion of thin-film coating between $\mathrm{PP}$ and $\mathrm{COC}$, which is critical for the structure and performance stability of the hybrid piezoelectrets. Fig. 3 shows the cross-section images of COC-coated PP films. In both cases, the COC was coated uniformly on the PPs. The average thickness of PP substrate layers was about $30 \mu \mathrm{m}$, and
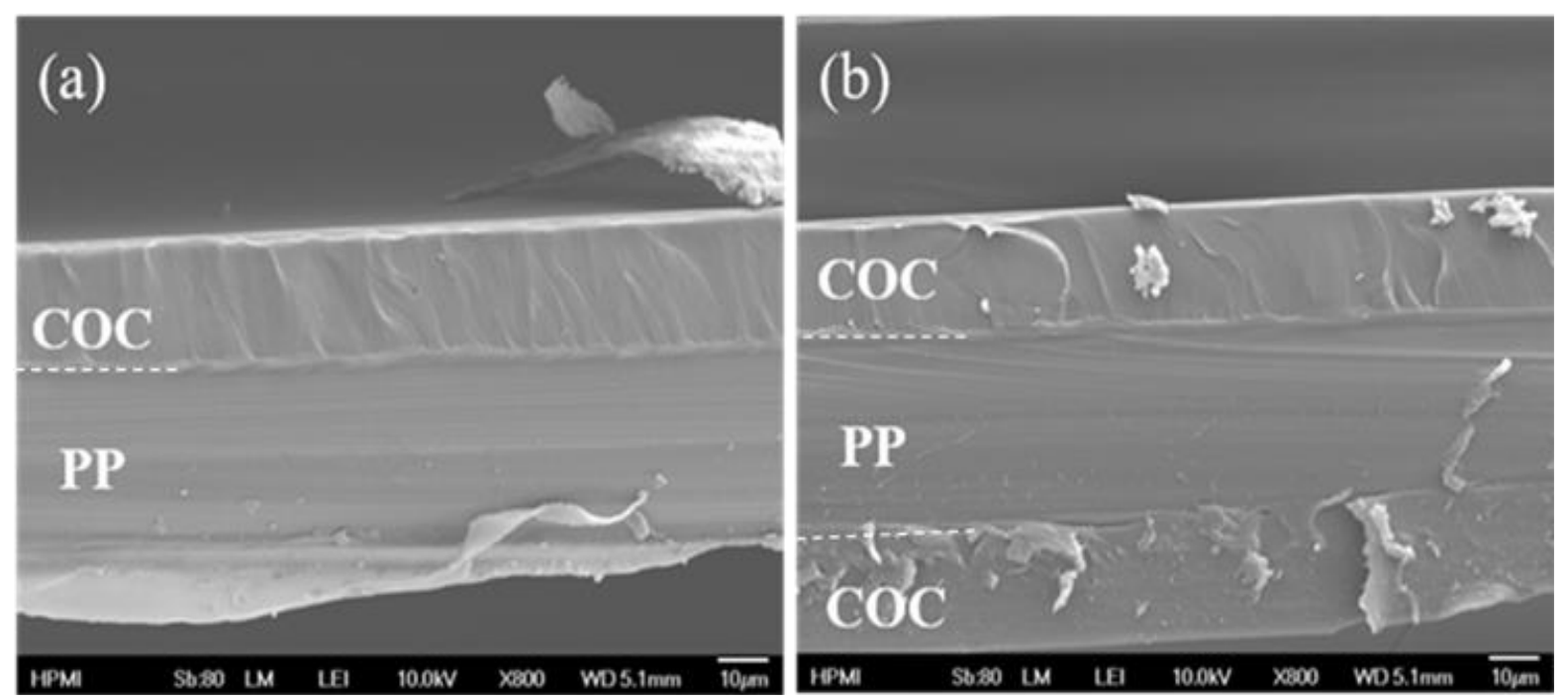

Fig. 3 SEM image of (a) single-side coated PP film and (b) double-side coated PP film. Dotted lines indicate the location of the bonding lines. Note that the irregular pale geometries are under the PP layer in 3a is a stretch PP region, an artifact caused by sample preparation.

the COC coating layer was about $15 \mu \mathrm{m}$. No void or gap was observed near the bond line, suggesting the process resulted in a good COC coating that adhered well with the substrate PPs.

\subsection{Piezoelectric activity}

The piezoelectric coefficient $\mathrm{d}_{33}$ of the hybrid COC/PP piezoelectric films was measured, and Fig. 4 shows the results. The piezoelectric coefficient $d_{33}$ was considerably higher than those reported in previous studies, ${ }^{[34]}$, exceeding $1000 \mathrm{pC} / \mathrm{N}$ at low pressures. $\mathrm{d}_{33}$ coefficient decreased with increasing pressure. This might result from the complex deformation characteristics of the sandwich structure ${ }^{15}$ and charge redistribution. Nevertheless, the piezoelectrets displayed respectable piezoelectric activity over the entire pressure range.

The piezoelectric coefficient $\mathrm{d}_{33}$ is dependent on both the electrical and mechanical properties of the piezoelectrets according to the following: ${ }^{[35]}$ 


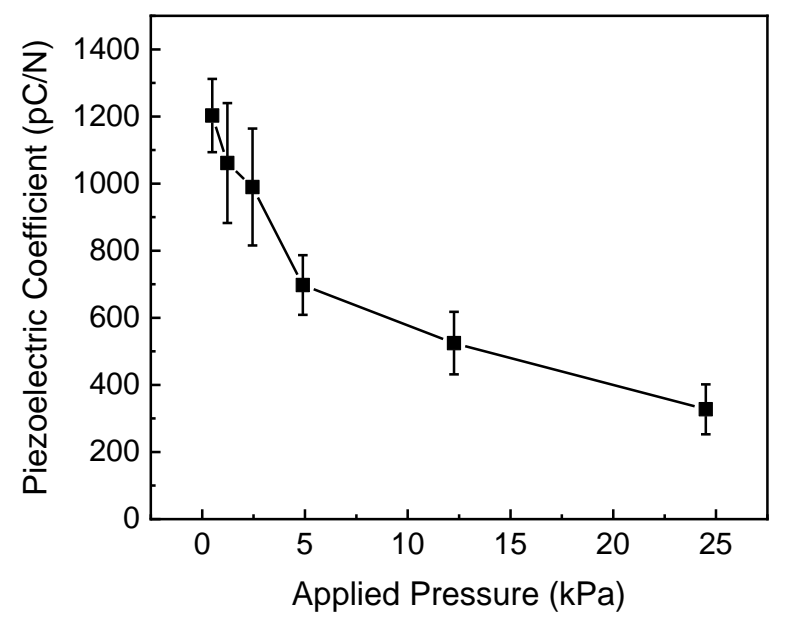

Fig. 4 Quasi-static piezoelectric $\mathrm{d}_{33}$ coefficient as a function of applied pressure of hybrid COC/PP assembled films.

$$
d_{33} \propto K \frac{\sigma_{\mathrm{eff}}}{E}
$$

where $d_{33}$ is the piezoelectric activity, $\mathrm{K}$ is a structure-related constant, $\sigma_{\text {eff }}$ is the effective polarization in the piezoelectrets, and $\mathrm{E}$ is the modulus of the materials. It follows that $\mathrm{d}_{33}$ is inversely proportional to the compression modulus of the materials. To improve the piezoelectric performance, many studies have attempted to reduce the effective modulus of the materials by inducing a porous structure ${ }^{[36-39]}$ In the current study, five layers of hybrid films were assembled to realize a
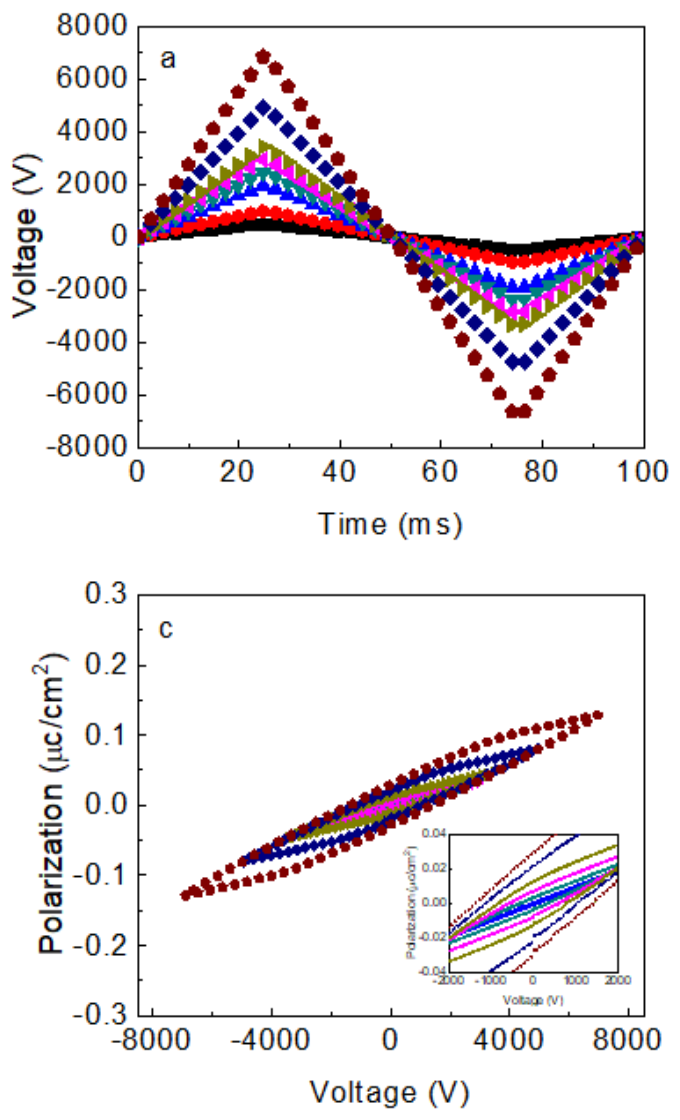

sandwich structure. This design principle was previously used for COC-based piezoelectrets. ${ }^{[15]}$ The such structure utilizes the large deflection in the individual film layer to achieve large overall compression displacement, thus achieving lower effective modulus and improving the piezoelectric activity. The design principle was also influential in significantly reducing the compressive modulus, and increasing the piezoelectric activity. The mechanism of the modulus reduction in the COC/PP hybrid piezoelectrets will be discussed in detail in section 3.4.

\subsection{Electrical hysteresis loop and remnant polarization}

To investigate the charging process and buildup of the "macrodipoles" in the inner voids of the piezoelectrets, electrical hysteresis loops measurements were conducted on both COC/PP hybrid piezoelectrets and COC piezoelectrets (for comparison). Fig. 5(a) shows the applied voltage profiles and Figs. 5(b) and 5(c) show the corresponding electric hysteresis loops of the two piezoelectrets. The quasi-permanent inducedcharge density was calculated from the hysteresis measurements, and Fig. 5(d) shows the results. For the hybrid COC/PP piezoelectrets, the onset of the electric-hysteresis loop, or minimum polarization voltage, appeared at around $1500 \mathrm{~V}$. The COC piezoelectrets had slightly higher critical breakdown voltage at about $2000 \mathrm{~V}$. For both piezoelectrets, the electric-hysteresis loops became more prominent upon increasing the charging voltage, leading to an increased quasi-
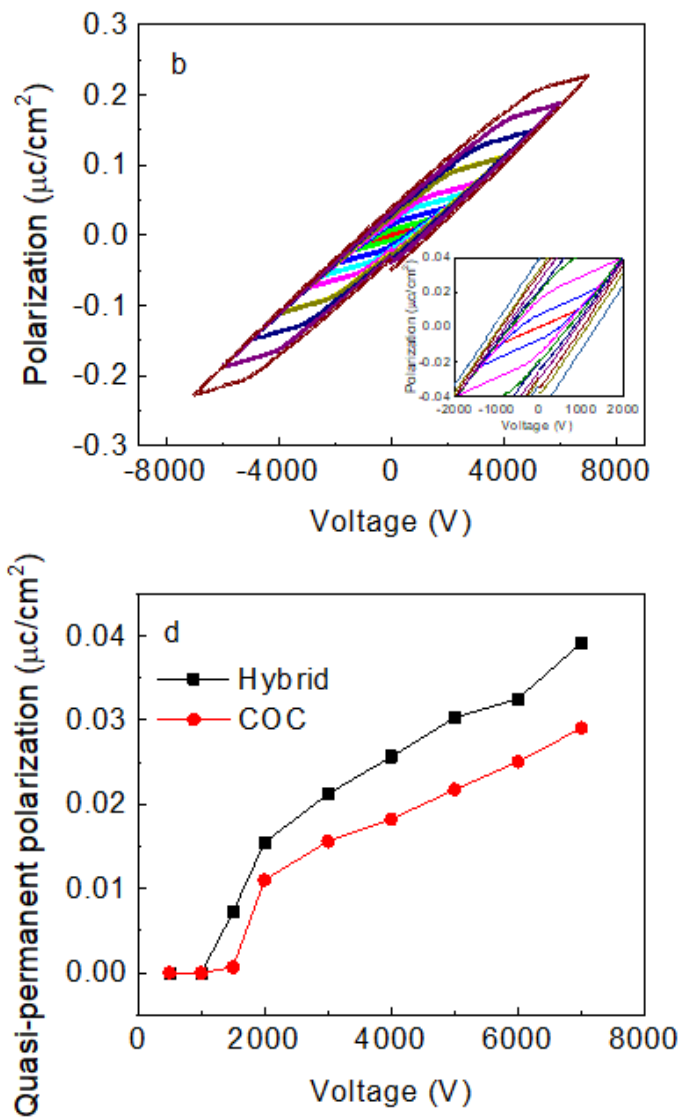

Fig. 5 (a) Applied voltage until $7000 \mathrm{~V}$ as a function of time, (b) quasi-static charge build of the hybrid COC/PP piezoelectrets and COC piezoelectrets, (c) hysteresis loop of hybrid COC/PP films, (d) comparison of quasi-static charge buildup in the artificial void. 
permanent charge density on the surfaces of the inner voids. Fig. 5 also shows that the induced-charge densities improved with the applied voltage beyond the critical values, increasing dielectric barrier discharge in the films. The induced-charge densities reached $0.038 \mu \mathrm{C} / \mathrm{cm}^{2}$ and $0.027 \mu \mathrm{C} / \mathrm{cm}^{2}$ for the hybrid $\mathrm{COC} / \mathrm{PP}$ piezoelectric film and $\mathrm{COC}$ piezoelectric film at a peak voltage of $7000 \mathrm{~V}$, respectively. The higher critical breakdown voltage and lower polarization and charge density at the same charging voltage for the COC piezoelectrets were due to its larger thickness, which required higher voltage to reach the same electrical field strength $(E=V / d, d$ is the thickness).

\subsection{Finite element analysis}

Although there is a great deal of piezoelectrets made from PP, the PP based piezoelectrets suffer low thermal stability and show poor piezoelectric performance in high-temperature environments. ${ }^{[16,40]}$ The COC/PP hybrid piezoelectrets address this shortcoming by using the significantly more thermally stable COC to store the charges while maintaining the overall flexibility enabled by PP. The piezoelectric coefficient $d_{33}$ of the piezoelectrets, is inversely proportional to the compression modulus of the materials. ${ }^{[29]}$ The current study achieved the modulus reduction by a sandwich structure design and implementation of anisotropic cavities in the hybrid piezoelectrets. Finite element analysis simulation on the fivelayer structure was conducted using COMSOL Multiphysics to provide insight into the deformation characteristics of the hybrid piezoelectrets. For comparison, both deformation of the hybrid COC/PP structure and pure COC were simulated. The model is based on a linear elastic constitutive equation throughout the domain. Figs. 6(a) and 6(b) show the unit structure of $\mathrm{COC} / \mathrm{PP}$ hybrid piezoelectrets and $\mathrm{COC}$ piezoelectrets, respectively. The actual geometric dimensions of the fabricated piezoelectrets were implemented in the model. Table 1 shows the properties of the material used for the simulation, and Table 2 shows the dimensions of the cavities. Fig. $6 \mathrm{c}$ shows the finite element mesh used in the simulation. Simulations were conducted using different cavity geometries. During the simulation, the bottom surface of the unit cell was selected as a fixed constraint, and the boundary load was applied on the top surface.

Figure 7 shows the results of cell deformations with the cell design strategy. The deformation within the structure varies (Fig. 7a). The most significant deformation occurred at the center of the artificial voids (cavities), and it gradually decreased toward the walls of the artificial voids. Note that the walls of the artificial voids were the pillars that cause the deflection of the top surface of the underneath artificial voids. The COC/PP hybrid piezoelectrets had a larger range of deflection than the COC piezoelectrets. Fig. $7 \mathrm{~b}$ plots the deformation in the thickness direction as a function of the applied pressure for the two piezoelectrets. COC/PP had substantially more significant deformation than $\mathrm{COC}$ piezoelectrets under the same pressure. Moreover, the effects of the aspect ratio of the cavities were investigated by varying the pore width from 1-3 mm while maintaining the same pore height of $1 \mathrm{~mm}$. Increasing the pore width led to increasing deflection. The structure with a $3 \mathrm{~mm}$ pore width, which was the one used to fabricate the piezoelectrets, produced the highest deflection. Using the theoretical thickness of the hybrid piezoelectrets of $270 \mu \mathrm{m}$ (calculated by summation of the thickness of the five layers), the strains under different pressures can be calculated. The effective modulus of the hybrid piezoelectrets with different pore aspect ratios was calculated. Table 2 shows the results.

Table 2. Effect of pore width on the apparent compressive modulus of the porous structure.

\begin{tabular}{cccccc}
\hline $\begin{array}{c}\text { Porous width } \\
(\mathrm{mm})\end{array}$ & Porosity & $\begin{array}{c}\text { Aspect } \\
\text { ratio }\end{array}$ & Ep $(\mathrm{kPa})$ & $\begin{array}{c}\text { Elastic } \\
\text { modulus* } \\
(\mathrm{kPa})\end{array}$ & $\begin{array}{c}\text { Modulus } \\
\text { reduced }\end{array}$ \\
\hline 1 & 0.22 & 0.06 & 4444 & 930 & $79.1 \%$ \\
2 & 0.30 & 0.03 & 3951 & 63 & $98.4 \%$ \\
3 & 0.33 & 0.02 & 3333 & 20 & $99.4 \%$ \\
\hline
\end{tabular}

* pore thickness is $0.06 \mathrm{~mm}$ in all cases.

**The elastic modulus was calculated by the FEA modeling of the COC/PP hybrid foam structures.

(a)

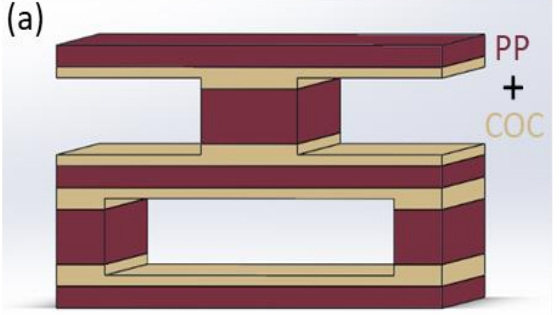

(b)

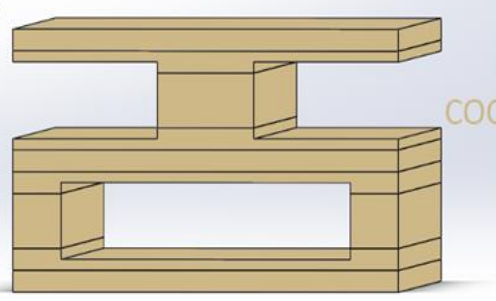

(c)

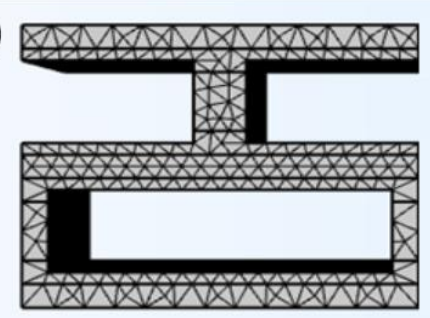

Fig. 6 Schematic unit cell of (a) hybrid COC/PP structure (b) neat COC structure (c) Finite element mesh used in simulation. For the hybrid piezoelectrets in (a), the thickness of the PP layer was $30 \mu \mathrm{m}$ and a COC coating layer of $15 \mu \mathrm{m}$. The thickness of the COC film in (b) was kept the same as model (a). 
(a)

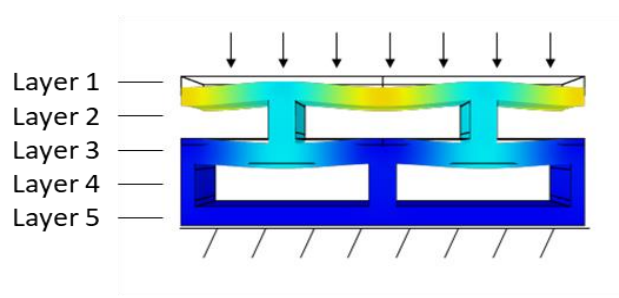

(b)

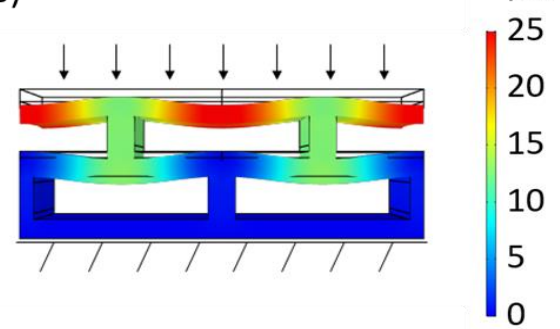

(c)

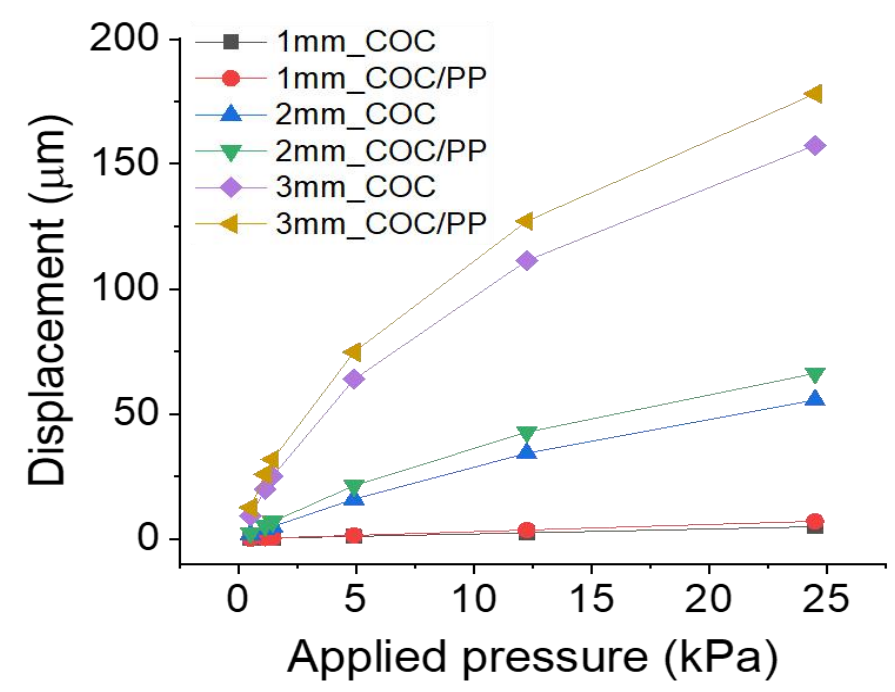

Fig. 7 Deformation of hybrid structures simulated by FEA with unit cell $3 \mathrm{~mm}$ porous width made by (a) COC at $1.1225 \mathrm{kPa}$ and (b) $\mathrm{COC} / \mathrm{PP}$ hybrid structure at $1.1225 \mathrm{kPa}$ (c) deformation comparison between pristine COC structure and COC/PP structure with the different cell design strategies (pore width of 1,2 and $3 \mathrm{~mm}$ ).

Classical mechanical theory on porous materials with anisotropic pores (voids) predicted that the material modulus is: ${ }^{[41]}$

$$
E_{p}=E_{s} \phi^{2} A
$$

where $E_{p}$ is the modulus of the porous material, $E_{S}$ is the modulus of the solid, $\phi$ is the porosity, and $A$ is the aspect ratio of the pores (voids). Both the porosity and aspect ratio could be calculated based on the geometry of the piezoelectrets structure. The porosity can be calculated by:

$$
\phi=\frac{V_{\text {void }}}{V_{\text {total }}}
$$

where $\mathrm{V}_{\text {void }}$ and $\mathrm{V}_{\text {total }}$ are the void volume and total volume, and the aspect ratio of the pores is defined as:

$$
A=\frac{h}{w} \text {. }
$$

where $h$ and $w$ are the height and width of the pore. For the fabricated $\mathrm{COC} / \mathrm{PP}$ piezoelectrets,

and

$$
h=60 \mu m
$$

Equations (2)-(6) yields,

$$
E_{p}=E_{s} \cdot\left(\frac{1}{3}\right)^{2} \cdot\left(\frac{0.06}{3}\right)=0.00222 E_{s}
$$

The hybrid piezoelectrets consist of PP and COC, and of the two materials, PP has a lower modulus. Using the low value of the modulus for PP, equation (7) leads to the lower bound of the compressive modulus if a porous material with the same porosity undergoes pure compression:

$$
\begin{gathered}
E_{p}=0.00222 \times 1.5 \times 10^{9} \mathrm{~Pa}= \\
3.333 \times 10^{6} \mathrm{~Pa}=3333 \mathrm{KPa}
\end{gathered}
$$

By comparison, the compressive modulus of the piezoelectrets with the sandwich structure was only $20 \mathrm{KPa}$ (Table 2). By implementing bending at the structure unit level, the modulus of the piezoelectrets was reduced by more than $99.4 \%$. The design strategy, which realizes large overall deformation through the deflection of the structural units, is highly effective in reducing the compression modulus to improve the piezoelectric activity of the piezoelectrets. Similar calculations were also performed with the cavity width of 1 and $2 \mathrm{~mm}$, and the results are also included in Table 2. The strategy is more effective when the anisotropy of the pore increases. Most importantly, such design is materials systems independent and thus can be a valuable principle in guiding the fabrication of piezoelectrets from other materials.

\subsection{Environmental stability of hybrid COC/PP piezoelectrets}

The environmental stability tests were performed under elevated temperatures and high relative humidity. Samples were conditioned at the prescribed temperature and humidity for a specified period (up to 200 hours), and their piezoelectric $\mathrm{d}_{33}$ was measured after that. Fig. 8 shows the results. The PP piezoelectrets rapidly lost their piezoelectric properties. After only 16 hours, the $\mathrm{d}_{33}$ decreases to almost zero at $70{ }^{\circ} \mathrm{C}$ under 


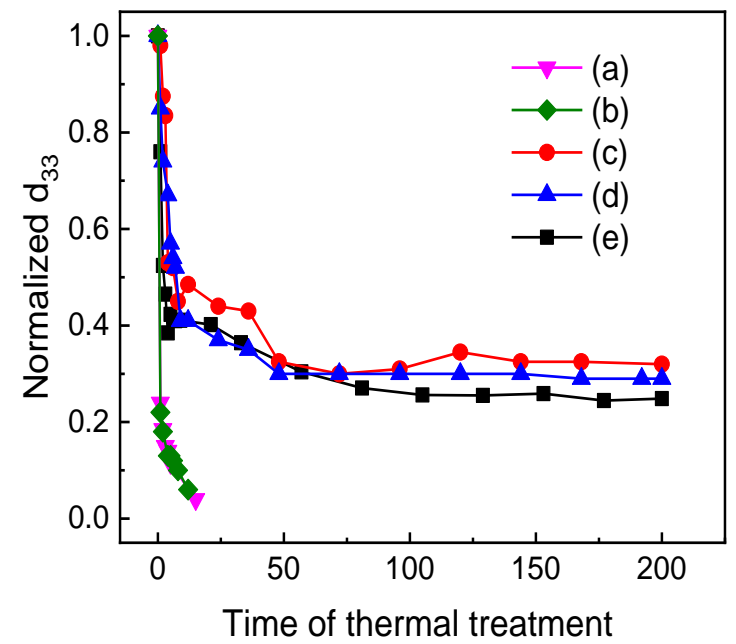

Fig. 8 Normalized piezoelectric activity decay of the PP piezoelectric films and hybrid $\mathrm{COC} / \mathrm{PP}$ piezoelectrets as a function of time (a) PP at $70{ }^{\circ} \mathrm{C}$ (b) $\mathrm{PP}$ at $70{ }^{\circ} \mathrm{C}$ at $\mathrm{RH} 90 \%$ (c) hybrid COC/PP at $70^{\circ} \mathrm{C}\left(\right.$ d) hybrid COC/PP at $70{ }^{\circ} \mathrm{C}$ and $\mathrm{RH} 90 \%$ (e) hybrid at $110^{\circ} \mathrm{C}$.

low and high humidity ((a) and (b)). This agrees with previous studies that PP piezoelectrets quickly lost their piezoelectric properties at temperatures above $70{ }^{\circ} \mathrm{C} \cdot{ }^{[13,42,43]}$ By comparison, the hybrid $\mathrm{COC} / \mathrm{PP}$ piezoelectrets showed significantly improved environmental stability. While the piezoelectric coefficient $\mathrm{d}_{33}$ also decreased during the initial annealing stage, the reduction was substantially lower. The $\mathrm{d}_{33}$ retained $50 \%$ of the initial value after 10 hours at $70{ }^{\circ} \mathrm{C}$. The piezoelectric coefficient $\mathrm{d}_{33}$ reached a stable value ( $\sim 35 \%$ of the initial value) after approximately 50 hours of annealing and remained stable after 200 hours. The hybrid piezoelectrets were also highly durable against humidity with only a minimum reduction of the activity under $90 \%$ relative humidity at $70{ }^{\circ} \mathrm{C}$. Even after annealing at $110{ }^{\circ} \mathrm{C}$ for 200 hours, the piezoelectrets retained substantial piezoelectric activity, with $\mathrm{d}_{33}$ about $25 \%$ of the initial value. The piezoelectricity in piezoelectrets arises from the stored charges and the resulting macroscopic dipoles. The stability of the charges is directly associated with the stability of the piezoelectrets. During the charging step, charges were generated by dielectric barrier breakdown and trapped in the dielectric polymer (COC and PP) to different energy levels. The charges with higher energy levels are easier to activate by thermal energy and gain sufficient mobility, leading to charge loss and reduction in piezoelectric activity. On the other hand, more deeply trapped charges have a considerably lower energy level and require much higher thermal energy (temperature) for activation. Unlike the PP electrets, in the COC/PP piezoelectrets, charges were stored in the $\mathrm{COC}$ layer and possessed superior thermal stability. This resulted in the vastly improved thermal stability of the hybrid COC/PP piezoelectrets.

\subsection{Sensor performance}

The force sensing capability of the COC/PP hybrid piezoelectrets was demonstrated. A cyclic compressive force was applied to the piezoelectret by a mechanical tester, and the sensor responses in open circuit voltage were measured using an electrometer. Fig. 9 shows the results. For clarity and ease of observation, signals of 10 cycles of the entire test $(1000$ cycles) were conducted. The piezoelectret sensor responded well to the applied force, and the sensor output voltage signal profile closely tracked that of the input force without noticeable delay (Figs. 9a and 9b). A similar pattern of the output signals under multiple test cycles also indicated the sensor could produce repeatable measurements, an essential requirement for sensing applications. Fig. 9c shows the output voltage signal as a function of the applied force. A linear relationship was observed. Overall, the piezoelectret sensor showed good response time, robust signal output, good repeatability, and linear output in response to the input signal. They may have potential in sensing applications under elevated temperatures and high humidity conditions.
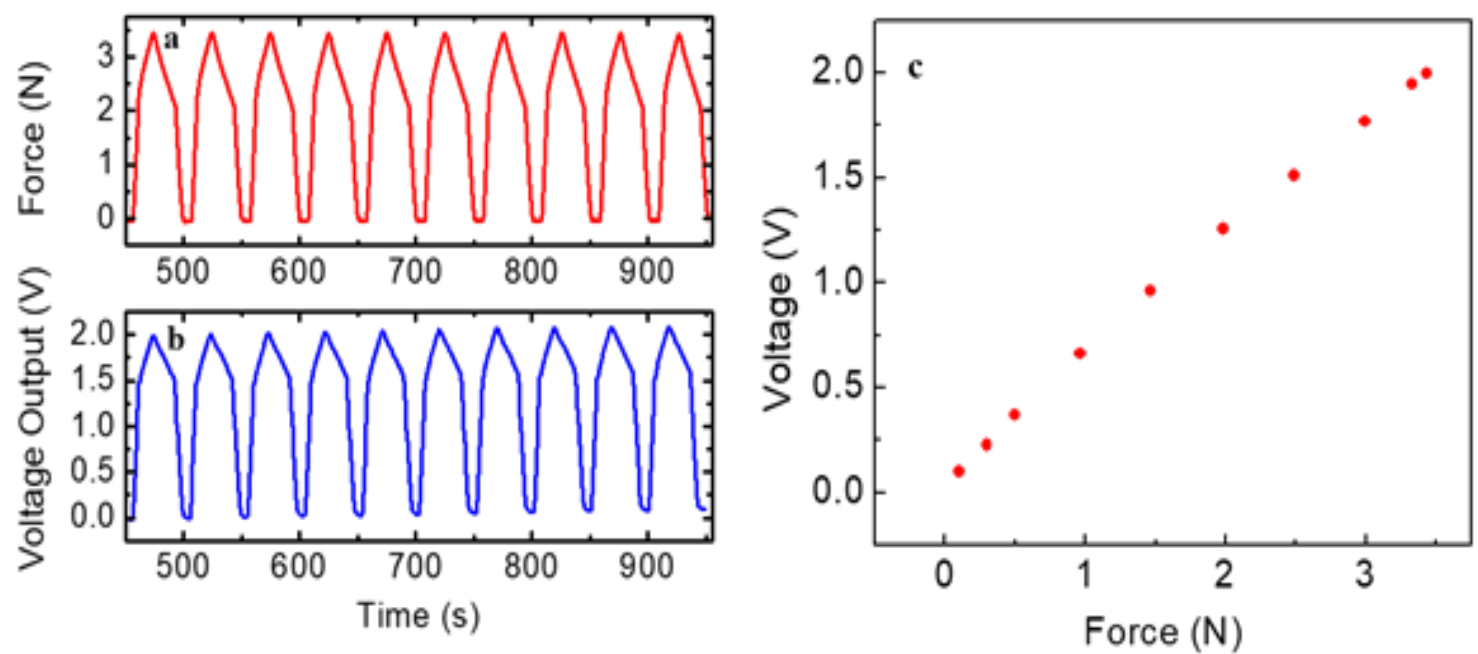

Fig. 9 Sensor performance of hybrid COC/PP piezoelectrets, (a) applied force versus time, (b) voltage output versus time, (c) relationship between applied force and voltage output. 


\section{Conclusions}

In this paper, flexible, low-cost, hybrid COC/PP piezoelectrets have been developed. The hybrid piezoelectrets had high piezoelectric activities, good flexibility, and excellent thermal and humidity stability, resulting from the synergy of the two polymers. Whereas PP provided excellent flexibility, COC enabled high thermal stability and good piezoelectric activity under high humidity. The high piezoelectric activity also benefited from the structure design in the study, which translated beam bending in the structure to the overall compression and significantly reduced compression modulus of the piezoelectrets. The fabrication process is potentially scalable. The piezoelectrets showed good performance in force sensing with fast response time, good signal linearity and repeatability, and reasonable robustness. With the excellent combination of piezoelectric activity, flexibility, and environmental stability, these thin-film-like hybrid piezoelectrets may have potential in many sensing applications, particularly wearable sensing and other situations where low-profile sensors are needed.

\section{Acknowledgments}

Assistance on the FEA analysis by Dr. Xuance Zhou of Illumina Inc. is acknowledged.

\section{Conflict of Interest}

There is no conflict of interest.

\section{Supporting Information}

Not applicable.

\section{References} 2004, 57, 37-43, doi: 10.1063/1.1688068.

[2] S. Bauer, IEEE Transactions on Dielectrics and Electrical Insulation, 2006, 13, 953-962 doi: 10.1109/TDEI.2006.247819.

[3] J. A. Gallego-Juarez, Journal of Physics E: Scientific Instruments, $\quad 1989, \quad \mathbf{2 2}, \quad 804-816$, doi: 10.1088/0022$3735 / 22 / 10 / 001$.

[4] H. Wei, H. Wang, Y. Xia, D. Cui, Y. Shi, M. Dong, C. Liu, T. Ding, J. Zhang, Y. Ma, N. Wang, Z. Wang, Y. Sun, R. Wei, Z. Guo, Journal of Materials Chemistry C, 2018, 6, 12446-12467, doi: $10.1039 / \mathrm{c} 8 \mathrm{tc} 04515 \mathrm{a}$.

[5] N. Wen, L. Zhang, D. Jiang, Z. Wu, B. Li, C. Sun, Z. Guo, Journal of Materials Chemistry A, 2020, 8, 25499-25527, doi: $10.1039 / \mathrm{d} 0 \mathrm{ta} 09556 \mathrm{~g}$.

[6] H. Wei, D. Cui, J. Ma, L. Chu, X. Zhao, H. Song, H. Liu, T. Liu, N. Wang, Z. Guo, Journal of Materials Chemistry A, 2017, 5, 1873-1894, doi: 10.1039/c6ta09726j.

[7] M. Lindner, H. Hoislbauer, R. Schwodiauer, S. BauerGogonea, S. Bauer, IEEE Transactions on Dielectrics and Electrical Insulation, 2004, 11, 255-263, doi: 10.1109/tdei.2004.1285895.

[8] K. Kirjavainen, Electromechanical film and procedure for manufacturing same, US Patent, 1987.
[1] S. Bauer, R. Gerhard-Multhaupt, G. M. Sessler, Physics Today,

[9] A. Mellinger, IEEE Transactions on Dielectrics and Electrical Insulation, 2003, 10, 842-861, doi: 10.1109/tdei.2003.1237333.

[10] A. Mellinger, M. Wegener, W. Wirges, R. R. Mallepally, R. Gerhard-Multhaupt, Ferroelectrics, 2006, 331, 189-199, doi: 10.1080/00150190600737933.

[11] G. C. Montanari, D. Fabiani, F. Ciani, A. Motori, M. Paajanen, R. Gerhard-Multhaupt, M. Wegener, IEEE Transactions on Dielectrics and Electrical Insulation, 2007, 14, 238-248, doi: 10.1109/tdei.2007.302892.

[12] Y. Wang, L. Wu, X. Zhang, IEEE Transactions on Dielectrics and Electrical Insulation, 2015, 22, 1349-1354, doi: 10.1109/tdei.2015.7116321.

[13] Z. Xiaoqing, H. Jinfeng, X. Zhongfu, Physica Scripta, 2007, T129, 274-277, doi: 10.1088/0031-8949/2007/T129/061.

[14] M. Sborikas, J. L. Ealo, M. Wegener, Sensors and Actuators A: Physical, 2016, 245, 1-9, doi: 10.1016/j.sna.2016.04.050.

[15] Y. Li, C. Zeng, Macromolecular Chemistry and Physics, 2013, 214, 2733-2738, doi: 10.1002/macp. 201300440.

[16] D. Pan, S. Luo, Y. Feng, X. Zhang, F. Su, H. Liu, C. Liu, X. Mai, N. Naik, Z. Guo, Composites Part B: Engineering, 2021, 222, 109039, doi: 10.1016/j.compositesb.2021.109039.

[17] Z. An, J. Yao, M. Mao, Y. Zhang, Z. Xia, Journal of Electrostatics, 2010, 68, 523-527, doi: 10.1016/j.elstat.2010.07.003.

[18] Z. An, M. Mao, J. Cang, Y. Zhang, F. Zheng, Journal of Applied Physics, 2012, 111, 024111, doi: 10.1063/1.3679576.

[19] J. Hillenbrand, G. M. Sessler, X. Zhang, Journal of Applied Physics, 2005, 98, 064105, doi: 10.1063/1.2058185.

[20] X. Zhang, G. M. Sessler, J. Hillenbrand, Journal of Electrostatics, 2007, $\quad \mathbf{6 5}, \quad 94-100, \quad$ doi: 10.1016/j.elstat.2006.07.006.

[21] L. Wu, X. Zhang, X. Zhang, Ceramics International, 2015, 41, S218-S222, doi: 10.1016/j.ceramint.2015.03.243.

[22] X. Zhang, G. Sessler, and J. Hillenbrand, 12th International Symposium on Electrets. (IEEE), 2005, 51-54.

[23] X. Zhang, X. Wang, J. Huang, Z. Xia, Journal of Materials Science, 2009, 44, 2459-2465, doi: 10.1007/s10853-009-3312-3.

[24] X. Zhang, J. Huang, X. Wang, Z. Xia, IEEE Transactions on Dielectrics and Electrical Insulation, 2010, 17, 1001-1007, doi: 10.1109/tdei.2010.5539667.

[25] X. Zhang, X. Wang, G. Cao, D. Pan, Z. Sun, Z. Xia, Ferroelectrics, 2010, 408, 55-61, doi: 10.1080/00150193.2010.485018.

[26] X. Zhang, X. Zhang, G. M. Sessler, X. Gong, Journal of Physics D: Applied Physics, 2014, 47, 015501, doi: 10.1088/0022-3727/47/1/015501.

[27] Z. Xia, R. Gerhard-Multhaupt, W. Künstler, A. Wedel, R. Danz, Journal of Physics D: Applied Physics, 1999, 32, L83-L85, doi: 10.1088/0022-3727/32/17/102.

[28] G. M. Yang, G. M. Sessler, and W. Hatke, 10th International Symposium on Electrets (ISE 10). Proceedings (Cat. No.99 CH36256), 1999, 317-320.

[29] A.-M. Savijarvi, M. Paajanen, E. Saarimaki, H. Minkkinen, 12th International Symposium on Electrets. (IEEE), 2005, 75-78. 
[30] H. Wang, Y. Li, X. Wang, Z. Liu, M. F. Ahmed, C. Zeng, Engineered Science, 2021, 16, 203-210, doi: 10.30919/es8d560.

[31] K. S. Ramadan, D. Sameoto, S. Evoy, Smart Materials and Structures, 2014, 23, 033001.

[32] D. Kline, R. Wool, Polymer Engineering Science, 1988, 28, 52-57, doi: 10.1002/pen.760280109.

[33] Z. Chen, C. Zeng, Z. Yao, K. Cao, Indian and Engineering Chemistry Research, 2013, 52, 9381-9396, doi: 10.1021/ie400833e.

[34] A. Mohebbi, F. Mighri, A. Ajji, D. Rodrigue, Advances in Polymer Technology, 2018, 37, 468-483, doi: 10.1002/adv.21686. [35] G. Sessler, J. Hillenbrand, 10th International Symposium on Electrets (ISE 10). Proceedings (Cat. No. 99 CH36256) (IEEE), 1999, 261-264.

[36] Altafim, Basso, Altafim, Lima, D. Aquino, IEEE Transactions on Dielectrics and Electrical Insulation, 2006, 13, 979-985, doi: 10.1109/tdei.2006.247822.

[37] R. Pisani Altafim, D. Rychkov, W. Wirges, R. Gerhard, H. Basso, R. Correa Altafim, M. Melzer, IEEE Transactions on Dielectrics and Electrical Insulation, 2012, 19, 1116-1123, doi: 10.1109/tdei.2012.6259978.

[38] T. Gómez Alvarez-Arenas, Sensors, 2013, 13, 5996-6013, doi: $10.3390 / \mathrm{s} 130505996$.

[39] X. Zhang, J. Hillenbrand, G. M. Sessler, Applied Physics A, 2006, 84, 139-142, doi: 10.1007/s00339-006-3573-5.

[40] E. Klimiec, B. Królikowski, M. Machnik, W. Zaraska, J. Dzwonkowski, Journal of Electronic Materials, 2015, 44, 22832291, doi: 10.1007/s11664-015-3719-3.

[41] L. J. Gibson, Mrs Bulletin, 2003, 28, 270-274, doi: 10.1557/mrs2003.79.

[42] X. Zhang, J. Huang, J. Chen, Z. Wan, S. Wang, Z. Xia, Applied Physics Letters, 2007, 91, 182901, doi: 10.1063/1.2803316.

[43] A. Mellinger, M. Wegener, W. Wirges, R. R. Mallepally, R. Gerhard-Multhaupt, Ferroelectrics, 2006, 331, 189-199, doi: 10.1080/00150190600737933.

Publisher's Note: Engineered Science Publisher remains neutral with regard to jurisdictional claims in published maps and institutional affiliations. 